IJMMS 25:4 (2001) 217-229

PII. S0161171201004707

http://ijmms.hindawi.com

(c) Hindawi Publishing Corp.

\title{
ON THE STABILITY OF THE QUADRATIC MAPPING IN NORMED SPACES
}

\author{
GWANG HUI KIM
}

(Received 3 December 1999 and in revised form 28 February 2000)

\begin{abstract}
The Hyers-Ulam stability, the Hyers-Ulam-Rassias stability, and also the stability in the spirit of Găvruța for each of the following quadratic functional equations $f(x+y)+f(x-y)=2 f(x)+2 f(y), f(x+y+z)+f(x-y)+f(y-z)+f(z-x)=$ $3 f(x)+3 f(y)+3 f(z), f(x+y+z)+f(x)+f(y)+f(z)=f(x+y)+f(y+z)+f(z+x)$ are investigated.
\end{abstract}

2000 Mathematics Subject Classification. Primary 39B52, 39B72, 39B82.

1. Introduction. The stability problem of functional equations was originally raised by Ulam [8] in 1940. He posed the following problem: under what conditions does there exist an additive mapping near an approximately additive mapping? In 1941, this problem was solved by Hyers [1] in the case of Banach space. Thereafter, we call that type the Hyers-Ulam stability. In 1978, Rassias [6] extended the Hyers-Ulam stability by considering variables. In 1994, it also has been generalized to the function case by Găvruța [3]. Throughout this paper, let $X$ and $Y$ be a real normed space and a real Banach space, respectively. Also $\mathbb{R}$ and $\mathbb{N}$ stand for the set of all real numbers and natural numbers, respectively.

The quadratic function $f(x)=x^{2}$ is a solution of each of the following functional equations

$$
\begin{gathered}
f(x+y)+f(x-y)=2 f(x)+2 f(y), \\
f(x+y+z)+f(x-y)+f(y-z)+f(z-x)=3 f(x)+3 f(y)+3 f(z), \\
f(x+y+z)+f(x)+f(y)+f(z)=f(x+y)+f(y+z)+f(z+x) .
\end{gathered}
$$

So, it is natural that each equation is called a quadratic functional equation. In particular, every solution of the "original" quadratic functional equation (1.1) is said to be a quadratic function.

For the quadratic functional equation some results are contained in $[1,2,4,7]$. Skof [7] and Cholewa [1] proved a Hyers-Ulam stability theorem of the quadratic functional equation (1.1) in different domains. Czerwik proved in [2] a Hyers-Ulam-Rassias stability for (1.1) which contains the following theorem as a particular case.

THEOREM 1.1. Let $\delta \geq 0$ be fixed. If $f: X \rightarrow Y$ satisfies the inequality

$$
\|f(x+y)+f(x-y)-2 f(x)-2 f(y)\| \leq \delta \quad \forall x \in X,
$$


then there exists a unique quadratic mapping $g: X \rightarrow Y$ such that

$$
\|g(x)-f(x)\| \leq \frac{\delta}{2} \quad \forall x \in X
$$

If, moreover, $f$ is measurable or $f(t x)$ is continuous in $t$ for each fixed $x \in X$, then $g(t x)=t^{2} g(x)$ for all $x \in X$ and $t \in \mathbb{R}$.

The stability in the sense of Rassias for (1.1) on a restricted domain and for (1.3) is proved by Jung [4]. In Section 2, the stability in the spirit of Găvruța of (1.1) (more generally, modified Hyers-Ulam-Rassias stability) is investigated. In Section 3, the HyersUlam stability, the Hyers-Ulam-Rassias stability, and the stability in the spirit of Găvruţa of (1.2) are investigated. In Section 4, the stability in the spirit of Găvruţa of (1.3) under the approximately even (or odd) condition is treated.

2. Stability in the spirit of Găvruța of (1.1). The stability of the quadratic functional equation (1.1) is proved under the spirit of Găvruţa. Let mappings $\varphi$ and $\Phi: X \times X \rightarrow[0, \infty)$ satisfy the inequality

$$
\Phi(x, y)=\frac{1}{6} \varphi(0,0)+\sum_{k=0}^{\infty} \frac{1}{4^{k+1}} \varphi\left(2^{k} x, 2^{k} y\right)<\infty \quad \forall x, y \in X
$$

By using an idea in Găvruța [3] we can prove the following results.

LEMmA 2.1. Assume that $f: X \rightarrow Y$ satisfies the inequality

$$
\|f(x+y)+f(x-y)-2 f(x)-2 f(y)\| \leq \varphi(x, y) \quad \forall x, y \in X .
$$

Then, for all $x \in X$ and $n \in \mathbb{N}$,

$$
\left\|f\left(2^{n} x\right)-4^{n} f(x)\right\| \leq \sum_{k=0}^{n-1} 4^{k} \frac{1}{2} \varphi(0,0)+\sum_{k=0}^{n-1} 4^{k} \varphi\left(2^{n-1-k} x, 2^{n-1-k} x\right) .
$$

Proof. Put $x=y=0$ in (2.2) and conclude that

$$
\|f(0)\| \leq \frac{1}{2} \varphi(0,0)
$$

For $x=y$ the inequality (2.2) again implies

$$
\|f(2 x)-4 f(x)\| \leq \frac{1}{2} \varphi(0,0)+\varphi(x, x) \quad \forall x \in X
$$

which proves the inequality (2.3) for $n=1$. For the induction, we assume that (2.3) 
holds for some $n \in \mathbb{N}$. Then, for any $x \in X$, we have, for $n+1$,

$$
\begin{aligned}
\left\|f\left(2^{n+1} x\right)-4^{n+1} f(x)\right\| \leq & \left\|f\left(2 \cdot 2^{n} x\right)-4 f\left(2^{n} x\right)\right\|+4\left\|f\left(2^{n} x\right)-4^{n} f(x)\right\| \\
\leq & \frac{1}{2} \varphi(0,0)+\varphi\left(2^{n} x, 2^{n} x\right) \\
& +4\left(\sum_{k=0}^{n-1} 4^{k} \frac{1}{2} \varphi(0,0)+\sum_{k=0}^{n-1} 4^{k} \varphi\left(2^{n-1-k} x, 2^{n-1-k} x\right)\right) \\
= & \sum_{k=0}^{n} 4^{k} \frac{1}{2} \varphi(0,0)+\sum_{k=0}^{n} 4^{k} \varphi\left(2^{n-k} x, 2^{n-k} x\right)
\end{aligned}
$$

which proves the inequality (2.3) for all natural $n$.

TheOREM 2.2. If $f: X \rightarrow Y$ satisfies the inequality (2.2), then there exists a unique quadratic mapping $g: X \rightarrow Y$ such that

$$
\|g(x)-f(x)\| \leq \Phi(x, x) \quad \forall x \in X .
$$

If, moreover, $f$ is measurable or $f(t x)$ is continuous in $t$ for each fixed $x \in X$, then $g(t x)=t^{2} g(x)$ for all $x \in X$ and $t \in \mathbb{R}$.

Proof. For any $x \in X$ and for every positive integer $n \in \mathbb{N}$, we define

$$
g_{n}(x)=4^{-n} f\left(2^{n} x\right) \text {. }
$$

From (2.3), we have, for $n>m$,

$$
\begin{aligned}
\left\|g_{n}(x)-g_{m}(x)\right\| & =4^{-n}\left\|f\left(2^{n-m} x \cdot 2^{m} x\right)-4^{n-m} f\left(2^{m} x\right)\right\| \\
& \leq 4^{-n}\left(\sum_{k=0}^{n-m-1} 4^{k} \frac{1}{2} \varphi(0,0)+\sum_{k=0}^{n-m-1} 4^{k} \varphi\left(2^{n-1-k} x, 2^{n-1-k} x\right)\right) \\
& =\sum_{k=0}^{n-m-1} 4^{-n+k} \frac{1}{2} \varphi(0,0)+\sum_{k=0}^{n-m-1} 4^{-n+k} \varphi\left(2^{n-1-k} x, 2^{n-1-k} x\right) \\
& =\sum_{k=m+1}^{n} 4^{-k} \frac{1}{2} \varphi(0,0)+\sum_{k=m+1}^{n} 4^{-k} \varphi\left(2^{k-1} x, 2^{k-1} x\right) .
\end{aligned}
$$

By (2.1), since the right-hand side of the above inequality tends to zero as $m$ tends to infinity, the sequence $\left\{g_{n}(x)\right\}$ is a Cauchy sequence for all $x \in X$. Since $Y$ is a Banach space, we define a function $g: X \rightarrow Y$ by

$$
\mathcal{g}(x)=\lim _{n \rightarrow \infty} g_{n}(x) \quad \forall x \in X .
$$

From the inequality (2.2), it follows that

$$
\begin{aligned}
\| g_{n}(x+y) & +g_{n}(x-y)-2 g_{n}(x)-2 g_{n}(y) \| \\
& =4^{-n}\left\|f\left(2^{n} x+2^{n} y\right)+f\left(2^{n} x-2^{n} y\right)-2 f\left(2^{n} x\right)-2 f\left(2^{n} y\right)\right\| \\
& \leq 4 \frac{1}{4^{n+1}} \varphi\left(2^{n} x, 2^{n} y\right)
\end{aligned}
$$


for all $x, y \in X$ and $n \in \mathbb{N}$. Therefore, by letting $n \rightarrow \infty$ in the last inequality we obtain (1.1) from (2.1). Moreover, from Lemma 2.1, for all $x \in X$ and $n \in \mathbb{N}$ we have the inequality:

$$
\begin{aligned}
\left\|g_{n}(x)-f(x)\right\| & =4^{-n}\left\|f\left(2^{n} x\right)-4^{n} f(x)\right\| \\
& \leq 4^{-n}\left(\sum_{k=0}^{n-1} 4^{k} \frac{1}{2} \varphi(0,0)+\sum_{k=0}^{n-1} 4^{k} \varphi\left(2^{n-1-k} x, 2^{n-1-k} x\right)\right) \\
& =\sum_{k=0}^{n-1} 4^{-n+k} \frac{1}{2} \varphi(0,0)+\sum_{k=0}^{n-1} 4^{-n+k} \varphi\left(2^{n-1-k} x, 2^{n-1-k} x\right) .
\end{aligned}
$$

Hence from (2.1) we see that (2.7) holds true.

If $h: X \rightarrow Y$ is another function which satisfies (1.1) and (2.7), since $g(0)=0=h(0)$, then by (1.1) we have

$$
g\left(2^{n} x\right)=4^{n} g(x), \quad h\left(2^{n} x\right)=4^{n} h(x)
$$

for all $x \in X$ and $n \in \mathbb{N}$. Hence, by (2.7) it follows that

$$
\begin{aligned}
\|g(x)-h(x)\| & =4^{-n}\left\|g\left(2^{n} x\right)-h\left(2^{n} x\right)\right\| \\
& \leq 4^{-n}\left(\left\|g\left(2^{n} x\right)-f\left(2^{n} x\right)\right\|+\left\|f\left(2^{n} x\right)-h\left(2^{n} x\right)\right\|\right) \\
& \leq \frac{2 \Phi\left(2^{n} x, 2^{n} x\right)}{4^{n}}
\end{aligned}
$$

for all $x \in X$ and $n \in \mathbb{N}$. By letting $n \rightarrow \infty$ in the preceding inequality, we immediately see the uniqueness of $g$. The proof of the last assertion in the theorem goes through in the same way as that of Theorem 1.1 (see [2, Theorem 1]).

NoTE. The last assertion in all results of this paper goes through in the same way as that of Theorem 1.1 (see [2, Theorem 1]).

The following corollary is the Hyers-Ulam stability of quadratic functional equation (1.1) which is the result of Skof [7] and Cholewa [1]. Applying Theorem 2.2 with $\varphi(x, y)=\delta$, we get the following corollary.

COROLlary 2.3. If $f: X \rightarrow Y$ satisfies the inequality

$$
\|f(x+y)+f(x-y)-2 f(x)-2 f(y)\| \leq \delta,
$$

then there exists a quadratic mapping $g: X \rightarrow Y$ satisfying (1.1) and such that

$$
\|g(x)-f(x)\| \leq \frac{\delta}{2} \quad \forall x \in X .
$$

If, moreover, $f$ is measurable or $f(t x)$ is continuous in $t$ for each fixed $x \in X$, then $g(t x)=t^{2} g(x)$ for all $x \in X$ and $t \in \mathbb{R}$.

The following theorem is the Hyers-Ulam-Rassias stability of quadratic functional equation (1.1). 
THEOREM 2.4. Let $X$ be a normed space and $Y$ a Banach space and let $\xi, \theta \geq 0$ and $p<2$ be given real numbers. Let $f: X \rightarrow Y$ be a function satisfying the inequality

$$
\|f(x+y)+f(x-y)-2 f(x)-2 f(y)\| \leq \xi+\theta\left(\|x\|^{p}+\|y\|^{p}\right) \quad \forall x, y \in X .
$$

Then there exists exactly one quadratic mapping $g: X \rightarrow Y$ such that

$$
\|g(x)-f(x)\| \leq \frac{1}{2} \xi+2\left(4-2^{p}\right)^{-1} \theta\|x\|^{p}, \quad x \in X .
$$

If, moreover, $f$ is measurable or $f(t x)$ is continuous in $t$ for each fixed $x \in X$, then $g(t x)=t^{2} g(x)$ for all $x \in X$ and $t \in \mathbb{R}$.

Theorem 2.4 is easily proved using Theorem 2.2.

COROLlary 2.5 [2, Theorem 1]. Let $X$ be a normed space and $Y$ a Banach space and let $\xi, \theta \geq 0$ and $p<2$ be given real numbers. Let $f: X \rightarrow Y$ be a function satisfying the inequality

$$
\|f(x+y)+f(x-y)-2 f(x)-2 f(y)\| \leq \xi+\theta\left(\|x\|^{p}+\|y\|^{p}\right) \quad \forall x, y \in X \backslash\{0\} .
$$

Then there exists exactly one quadratic mapping $g: X \rightarrow Y$ such that

$$
\|g(x)-f(x)\| \leq \frac{\xi+\|f(0)\|}{3}+2\left(4-2^{p}\right)^{-1} \theta\|x\|^{p}, \quad x \in X \backslash\{0\} .
$$

If, moreover, $f$ is measurable or $f(t x)$ is continuous in $t$ for each fixed $x \in X$, then $g(t x)=t^{2} g(x)$ for all $x \in X$ and $t \in \mathbb{R}$.

Proof. For $x=y \in X \backslash\{0\}$ the inequality (2.2) in Lemma 2.1 implies

$$
\|f(2 x)-4 f(x)\| \leq\|f(0)\|+\varphi(x, x) \quad \forall x \in X \backslash\{0\},
$$

then we have

$$
\left\|f\left(2^{n} x\right)-4^{n} f(x)\right\| \leq \sum_{k=0}^{n-1} 4^{k}\|f(0)\|+\sum_{k=0}^{n-1} 4^{k} \varphi\left(2^{n-1-k} x, 2^{n-1-k} x\right) \quad \forall x \in X \backslash\{0\} .
$$

By applying Theorem 2.2 with $\varphi(x, y)=\theta\left(\|x\|^{p}+\|y\|^{p}\right)$ for $p<2$, the proof of the corollary is complete.

3. Three types stability of (1.2). In this section, we investigate the Hyers-Ulam stability, Hyers-Ulam-Rassias stability, and the stability in the spirit of Găvruța for (1.2).

LEMмA 3.1. Assume that $f: X \rightarrow Y$ satisfies the inequality

$$
\|f(x+y+z)+f(x-y)+f(y-z)+f(z-x)-3 f(x)-3 f(y)-3 f(z)\| \leq \delta
$$

for all $x, y, z \in X$ and $\delta \geq 0$. Then for $x \in X$ and $n \in \mathbb{N}$,

$$
\left\|f\left(3^{n} x\right)-3^{2 n} f(x)\right\| \leq \frac{8}{5} \delta \sum_{k=1}^{n} 3^{2(k-1)} .
$$


Proof. Put $x=y=z=0$ in (3.1) and conclude that

$$
\|f(0)\| \leq \frac{\delta}{5} \text {. }
$$

For $x=y=z$, the inequality (3.1) again implies

$$
\left\|f(3 x)-3^{2} f(x)\right\| \leq \delta+3\|f(0)\| \leq \frac{8}{5} \delta
$$

which proves the inequality (3.2) for $n=1$. For the induction, we assume that (3.2) holds for some $n \in \mathbb{N}$. Then, for any $x \in X$, by (3.4) we have, for $n+1$,

$$
\begin{aligned}
& \left\|f\left(3^{n+1} x\right)-3^{2(n+1)} f(x)\right\| \\
& \quad \leq\left\|f\left(3 \cdot 3^{n} x\right)-3^{2} f\left(3^{n} x\right)\right\|+3^{2}\left\|f\left(3^{n} x\right)-3^{2 n} f(x)\right\| \\
& \quad \leq \frac{8}{5} \delta+3^{2}\left(\frac{8}{5} \delta \sum_{k=1}^{n} 3^{2(k-1)}\right)=\frac{8}{5} \delta\left(1+\sum_{k=2}^{n+1} 3^{2(k-1)}\right)=\frac{8}{5} \delta \sum_{k=1}^{n+1} 3^{2(k-1)}
\end{aligned}
$$

which proves the inequality (3.2) for all natural $n$.

THEOREM 3.2. Assume that a mapping $f: X \rightarrow Y$ satisfies the inequality (3.1). Then there exists a unique quadratic mapping $g: X \rightarrow Y$ satisfying (1.2) and the inequality

$$
\|g(x)-f(x)\| \leq \frac{\delta}{5} \quad \forall x \in X .
$$

If, moreover, $f$ is measurable or $f(t x)$ is continuous in $t$ for each fixed $x \in X$, then $g(t x)=t^{2} g(x)$ for all $x \in X$ and $t \in \mathbb{R}$.

Proof. For any $x \in X$ and for every positive integer $n \in \mathbb{N}$, we define

$$
g_{n}(x)=3^{-2 n} f\left(3^{n} x\right) .
$$

From (3.4), we have

$$
\left\|g_{n+1}(x)-g_{n}(x)\right\|=3^{-2(n+1)}\left\|f\left(3 \cdot 3^{n} x\right)-3^{2} f\left(3^{n} x\right)\right\| \leq 3^{-2(n+1)} \frac{8}{5} \delta
$$

for all $n>N$ and for all $x \in X$. Hence we have, for $n \geq m$,

$$
\left\|g_{n}(x)-g_{m}(x)\right\| \leq \sum_{j=m}^{n-1}\left\|g_{j+1}(x)-g_{j}(x)\right\| \leq \frac{8}{5} \delta \sum_{j=m}^{n-1} 3^{-2(j+1)} \longrightarrow 0
$$

for all $n>N$ and for all $x \in X$ as $m \rightarrow \infty$. We see that the sequence $\left\{g_{n}(x)\right\}$ is a Cauchy sequence. Hence, we can define a function $g: X \rightarrow Y$ by

$$
g(x)=\lim _{n \rightarrow \infty} g_{n}(x) \quad \forall x \in X .
$$

Then for all $x, y, z \in X$ and $n \in \mathbb{N}$, we have, from (3.1),

$$
\begin{gathered}
\left\|g_{n}(x+y+z)+g_{n}(x-y)+g_{n}(y-z)+g_{n}(z-x)-3 g_{n}(x)-3 g_{n}(y)-3 g_{n}(z)\right\| \\
=3^{-2 n} \| f\left(3^{n}(x+y+z)\right)+f\left(3^{n}(x-y)\right)+f\left(3^{n}(y-z)\right)+f\left(3^{n}(z-x)\right) \\
-3 f\left(3^{n} x\right)-3 f\left(3^{n} y\right)-3 f\left(3^{n} z\right) \| \leq \frac{\delta}{3^{2 n}} .
\end{gathered}
$$


Letting $n$ tend to infinity, we obtain (1.2). Moreover, from Lemma 3.1, we have

$$
\begin{aligned}
\left\|g_{n}(x)-f(x)\right\| & =3^{-2 n}\left\|f\left(3^{n} x\right)-3^{2 n} f(x)\right\| \\
& \leq 3^{-2 n} \frac{8}{5} \delta \sum_{k=1}^{n} 3^{2(k-1)}=\frac{8}{5} \delta \sum_{k=1}^{n} 3^{-2 k}
\end{aligned}
$$

for all $x, y \in X$ and $n \in \mathbb{N}$. By letting $n$ tend to infinity, we obtain the inequality (3.6).

The proof of the uniqueness is the same way as that of Theorem 2.2 by applying $g\left(3^{n} x\right)=3^{2 n} g(x)$ and $h\left(3^{n} x\right)=3^{2 n} h(x)$. Hence, the proof is complete.

Let the mappings $\varphi$ and $\Phi: X \times X \times X \rightarrow[0, \infty)$ satisfy the inequality

$$
\Phi(x, y, z)=\frac{3}{130} \varphi_{0}+\sum_{k=1}^{\infty} 3^{-3 k} \varphi\left(3^{k-1} x, 3^{k-1} y, 3^{k-1} z\right)<\infty .
$$

For simplicity of calcuation in this section, we use the notation $\varphi_{x}=\varphi(x, x, x)$.

LEMmA 3.3. Assume that $f: X \rightarrow Y$ satisfies the inequality

$$
\|f(x+y+z)+f(x-y)+f(y-z)+f(z-x)-3 f(x)-3 f(y)-3 f(z)\| \leq \varphi(x, y, z)
$$

for all $x, y, z \in X$. It then holds that for all $x \in X$ and for all $n \in \mathbb{N}$,

$$
\left\|f\left(3^{n} x\right)-3^{3 n} f(x)\right\| \leq \frac{3}{5} \varphi_{0} \sum_{k=1}^{n} 3^{3(k-1)}+\sum_{k=1}^{n} 3^{3(n-k)} \varphi_{3^{k-1} x} .
$$

Proof. Put $x=y=z=0$ in (3.14) and conclude that

$$
\|f(0)\| \leq \frac{1}{5} \varphi_{0}
$$

For $x=y=z$, the inequality (3.14) again implies

$$
\left\|f(3 x)-3^{3} f(x)\right\| \leq 3\|f(0)\|+\varphi_{x} \leq \frac{3}{5} \varphi_{0}+\varphi_{x}
$$

which proves the inequality (3.15) for $n=1$. For the induction, we assume that (3.15) holds for some $n \in \mathbb{N}$ and for all $x \in X$. Then by (3.17) we have, for $n+1$,

$$
\begin{aligned}
\left\|f\left(3^{n+1} x\right)-3^{3(n+1)} f(x)\right\| & \leq\left\|f\left(3 \cdot 3^{n} x\right)-3^{3} f\left(3^{n} x\right)\right\|+3^{3}\left\|f\left(3^{n} x\right)-3^{3 n} f(x)\right\| \\
& \leq \frac{3}{5} \varphi_{0}+\varphi_{3^{n} x}+3^{3}\left(\frac{3}{5} \varphi_{0} \sum_{k=1}^{n} 3^{3(k-1)}+\sum_{k=1}^{n} 3^{3(n-k)} \varphi_{3^{k-1} x}\right) \\
& =\frac{3}{5} \varphi_{0} \sum_{k=1}^{n+1} 3^{3(k-1)}+\sum_{k=1}^{n+1} 3^{3(n-k)} \varphi_{3^{k-1} x}
\end{aligned}
$$

which proves the inequality (3.15) for all natural $n$.

THEOREM 3.4. Assume that a mapping $f: X \rightarrow Y$ satisfies the equality (3.14). Then there exists a unique quadratic mapping $g: X \rightarrow Y$ that satisfies (1.2) and the inequality

$$
\|g(x)-f(x)\| \leq \Phi_{x} \quad \forall x \in X
$$


If, moreover, $f$ is measurable or $f(t x)$ is continuous in $t$ for each fixed $x \in X$, then $g(t x)=t^{2} g(x)$ for all $x \in X$ and $t \in \mathbb{R}$.

PRoof. For every positive integer $n$, we define

$$
g_{n}(x)=3^{-3 n} f\left(3^{n} x\right) \quad \forall x \in X .
$$

By (3.17), we have

$$
\begin{aligned}
\left\|g_{n+1}(x)-g_{n}(x)\right\| & =3^{-3(n+1)}\left\|f\left(3 \cdot 3^{n} x\right)-3^{3} f\left(3^{n} x\right)\right\| \\
& \leq 3^{-3(n+1)} \frac{3}{5} \varphi_{0}+3^{-3(n+1)} \varphi_{3^{n} x}
\end{aligned}
$$

for all $n \in \mathbb{N}$ and for all $x \in X$. Hence by (3.21) we have, for $n \geq m$,

$$
\begin{aligned}
\left\|g_{n}(x)-g_{m}(x)\right\| & \leq \sum_{j=m}^{n-1}\left\|g_{j+1}(x)-g_{j}(x)\right\| \\
& \leq \frac{3}{5} \varphi_{0} \sum_{j=m}^{n-1} 3^{-3(j+1)}+\sum_{j=m}^{n-1} 3^{-3(j+1)} \varphi_{3^{j} x}
\end{aligned}
$$

for all $x \in X$. By (3.13) since the right-hand side of the preceding inequality tends to zero as $m$ tends to infinity, we see that the sequence $\left\{g_{n}(x)\right\}$ is a Cauchy sequence. Hence we can define a function $g: X \rightarrow Y$ by

$$
g(x)=\lim _{n \rightarrow \infty} g_{n}(x) \quad \forall x \in X .
$$

Then for all $x, y, z \in X$ and $n \in \mathbb{N}$, we have

$$
\begin{gathered}
\left\|g_{n}(x+y+z)+g_{n}(x-y)+g_{n}(y-z)+g_{n}(z-x)-3 g_{n}(x)-3 g_{n}(y)-3 g_{n}(z)\right\| \\
=3^{-3 n} \| f\left(3^{n}(x+y+z)\right)+f\left(3^{n}(x-y)\right)+f\left(3^{n}(y-z)\right)+f\left(3^{n}(z-x)\right) \\
-3 f\left(3^{n} x\right)-3 f\left(3^{n} y\right)-3 f\left(3^{n} z\right) \| \leq \frac{\varphi\left(3^{n} x, 3^{n} y, 3^{n} z\right)}{3^{3 n}} .
\end{gathered}
$$

Letting $n$ tend to infinity, we obtain (1.2). Moreover, from Lemma 3.3, we have

$$
\begin{aligned}
\left\|g_{n}(x)-f(x)\right\| & =3^{-3 n}\left\|f\left(3^{n} x\right)-3^{3 n} f(x)\right\| \\
& \leq 3^{-3 n}\left(\frac{3}{5} \varphi_{0} \sum_{k=1}^{n} 3^{3(k-1)}+\sum_{k=1}^{n} 3^{3(n-k)} \varphi_{3^{k-1} x}\right) \\
& =\frac{3}{5} \varphi_{0} \sum_{k=1}^{n} 3^{-3(n-k+1)}+\sum_{k=1}^{n} 3^{-3 k} \varphi_{3^{k-1} x} \\
& =\frac{3}{130}\left(1-\frac{1}{3^{3 n}}\right) \varphi_{0}+\sum_{k=1}^{n} 3^{-3 k} \varphi_{3^{k-1} x}
\end{aligned}
$$

for all $x \in X$ and $n \in \mathbb{N}$. From (3.13) as $n$ tend to infinity we obtain the inequality (3.19).

The proof of the uniqueness is the same way as that of Theorem 2.2 by applying $g\left(3^{n} x\right)=3^{2 n} g(x)$ and $h\left(3^{n} x\right)=3^{2 n} h(x)$. Hence, the proof is complete. 
The following theorem is the Hyers-Ulam-Rassias stability of quadratic functional equation (1.2).

THEOREM 3.5. Assume that a mapping $f: X \rightarrow Y$ satisfies the equality

$$
\begin{aligned}
\| f(x+y+z)+f(x-y)+f(y-z)+f(z-x)-3 f(x) & -3 f(y)-3 f(z) \| \\
& \leq \theta\left(\|x\|^{p}+\|y\|^{p}+\|z\|^{p}\right)
\end{aligned}
$$

for all $x, y, z \in X$ and $p<3$. Then there exists a unique quadratic mapping $g: X \rightarrow Y$ satisfies (1.2) and the inequality

$$
\|g(x)-f(x)\| \leq 3\left|3^{3}-3^{p}\right|^{-1} \theta\|x\|^{p} \quad \forall x \in X .
$$

If, moreover, $f$ is measurable or $f(t x)$ is continuous in $t$ for each fixed $x \in X$, then $g(t x)=t^{2} g(x)$ for all $x \in X$ and $t \in \mathbb{R}$.

Proof. Apply Theorem 3.2, with $\varphi(x, y, z)=\theta\left(\|x\|^{p}+\|y\|^{p}+\|z\|^{p}\right)$, and (3.13). Then we obtain

$$
\begin{aligned}
\Phi(x, x, x) & =\sum_{k=1}^{\infty} 3^{-3 k}\left[\theta\left(\left\|3^{k-1} x\right\|^{p}+\left\|3^{k-1} x\right\|^{p}+\left\|3^{k-1} x\right\|^{p}\right)\right] \\
& =3 \theta\|x\|^{p} \sum_{k=1}^{\infty} 3^{-3 k} 3^{(k-1) p}=3\left(3^{3}-3^{p}\right)^{-1} \theta\|x\|^{p} .
\end{aligned}
$$

4. Stability in the spirit of Găvruța of (1.3). In this section, the stability of another quadratic (1.3) is investigated under the spirit of Găvruța. The Hyers-Ulam-Rassias stability of (1.3) can be found in [4].

Let the mappings $\varphi$ and $\Phi: X \times X \times X \rightarrow[0, \infty)$ satisfy the inequality

$$
\begin{aligned}
\Phi(x, y, z)= & 2 \varphi(0,0,0)+\sum_{k=0}^{\infty} \frac{2^{k}+1}{2^{2 k+1}} \varphi\left(2^{k-1} x, 2^{k-1} y,-2^{k-1} z\right) \\
& +\sum_{k=0}^{\infty} \frac{2^{k}-1}{2^{2 k+1}} \varphi\left(-2^{k-1} x,-2^{k-1} y, 2^{k-1} z\right)<\infty \quad \forall x \in X .
\end{aligned}
$$

For simplicity of calculation in this section, we use the notation $\varphi_{x}=\varphi(x, x,-x)$.

LEMMA 4.1. Assume that a mapping $f: X \rightarrow Y$ satisfies the following inequality:

$$
\|f(x+y+z)+f(x)+f(y)+f(z)-f(x+y)-f(y+z)-f(z+x)\| \leq \varphi(x, y, z)
$$

for all $x, y, z \in X$. It then holds that

$$
\begin{aligned}
\| f(x) & -\frac{2^{n}+1}{2^{2 n+1}} f\left(2^{n} x\right)+\frac{2^{n}-1}{2^{2 n+1}} f\left(-2^{n} x\right) \| \\
& \leq \sum_{k=1}^{n}\left(\frac{1}{2^{k-1}} \varphi_{0}+\frac{2^{k}+1}{2^{2 k+1}} \varphi_{2^{k-1} x}+\frac{2^{k}-1}{2^{2 k+1}} \varphi_{-2^{k-1} x}\right)
\end{aligned}
$$

for all $x, y, z \in X$ and $n \in \mathbb{N}$. 
Proof. Put $x=y=z=0$ in (4.2) and conclude that $\|f(0)\| \leq \varphi_{0}$. And also putting $x=y=-z$ in (4.2) yields

$$
\|3 f(x)+f(-x)-f(2 x)\| \leq 2 \varphi_{0}+\varphi_{x} .
$$

Substitute $-x$ for $x$ in (4.4), we obtain

$$
\|3 f(-x)+f(x)-f(-2 x)\| \leq 2 \varphi_{0}+\varphi_{-x} .
$$

We use induction on $n$ to prove our lemma. By (4.4) and (4.5), we have

$$
\begin{aligned}
\| f(x) & -\frac{3}{8} f(2 x)+\frac{1}{8} f(-2 x) \| \\
& \leq \frac{3}{8}\|3 f(x)+f(-x)-f(2 x)\|+\frac{1}{8}\|3 f(-x)+f(x)-f(-2 x)\| \\
& \leq \frac{3}{8}\left(2 \varphi_{0}+\varphi_{x}\right)+\frac{1}{8}\left(2 \varphi_{0}+\varphi_{-x}\right)=\varphi_{0}+\frac{3}{8} \varphi_{x}+\frac{1}{8} \varphi_{-x}
\end{aligned}
$$

which proves the validity of the inequality (4.3) for the case $n=1$. Now assume that the inequality (4.3) holds true for some $n \in \mathbb{N}$. By using (4.4) and (4.5), we have the following relation:

$$
\begin{aligned}
\| f(x)- & \frac{2^{n+1}+1}{2^{2 n+3}} f\left(2^{n+1} x\right)+\frac{2^{n+1}-1}{2^{2 n+3}} f\left(-2^{n+1} x\right) \| \\
\leq & \left\|f(x)-\frac{2^{n}+1}{2^{2 n+1}} f\left(2^{n} x\right)+\frac{2^{n}-1}{2^{2 n+1}} f\left(-2^{n} x\right)\right\| \\
& +\frac{2^{n+1}+1}{2^{2 n+3}}\left\|3 f\left(2^{n} x\right)+f\left(-2^{n} x\right)-f\left(2^{n+1} x\right)\right\| \\
& +\frac{2^{n+1}-1}{2^{2 n+3}}\left\|3 f\left(-2^{n} x\right)+f\left(2^{n} x\right)-f\left(-2^{n+1} x\right)\right\| \\
\leq & \sum_{k=1}^{n}\left(\frac{1}{2^{k-1}} \varphi_{0}+\frac{2^{k}+1}{2^{2 k+1}} \varphi_{2^{k-1} x}+\frac{2^{k}-1}{2^{2 k+1}} \varphi_{-2^{k-1} x}\right) \\
& +\frac{2^{n+1}+1}{2^{2 n+3}}\left(2 \varphi_{0}+\varphi_{2^{n} x}\right)+\frac{2^{n+1}-1}{2^{2 n+3}}\left(2 \varphi_{0}+\varphi_{-2^{n} x}\right) \\
= & \sum_{k=1}^{n+1}\left(\frac{1}{2^{k-1}} \varphi_{0}+\frac{2^{k}+1}{2^{2 k+1}} \varphi_{2^{k-1} x}+\frac{2^{k}-1}{2^{2 k+1}} \varphi_{-2^{k-1} x}\right)
\end{aligned}
$$

which proves the inequality (4.3) for $n+1$.

THEOREM 4.2. Assume that a mapping $f: X \rightarrow Y$ satisfies the inequalities (4.2) and

$$
\|f(x)-f(-x)\| \leq \theta
$$

for $\theta \geq 0$ and for all $x, y, z \in X$. Then there exists a unique quadratic mapping $g: X \rightarrow Y$ satisfying (1.3) such that

$$
\|g(x)-f(x)\| \leq \Phi(x) \quad \forall x \in X .
$$

If, moreover, $f$ is measurable or $f(t x)$ is continuous in $t$ for each fixed $x \in X$, then $g(t x)=t^{2} g(x)$ for all $x \in X$ and $t \in \mathbb{R}$. 
Proof. For any $x \in X$ and for every positive integer $n$, we define

$$
g_{n}(x)=2^{-2 n} f\left(2^{n} x\right) .
$$

From (4.4) and (4.8), we have

$$
\begin{aligned}
\| g_{n+1} & (x)-g_{n}(x) \| \\
& =2^{-2(n+1)}\left\|f\left(2 \cdot 2^{n} x\right)-2^{2} f\left(2^{n} x\right)\right\| \\
& \leq 2^{-2(n+1)}\left(\left\|3 f\left(2^{n} x\right)+f\left(-2^{n} x\right)-f\left(2 \cdot 2^{n} x\right)\right\|+\left\|f\left(2^{n} x\right)-f\left(-2^{n} x\right)\right\|\right) \\
& \leq 2^{-2(n+1)}\left(2 \varphi_{0}+\varphi_{2^{n} x}+\theta\right)
\end{aligned}
$$

for all $x \in X$ and for all $n \in \mathbb{N}$. Therefore we have, for $n \geq m$,

$$
\begin{aligned}
\left\|g_{n}(x)-g_{m}(x)\right\| & \leq \sum_{j=m}^{n-1}\left\|g_{j+1}(x)-g_{j}(x)\right\| \\
& \leq \sum_{j=m}^{n-1} 2^{-2(j+1)}\left(2 \varphi_{0}+\varphi_{2^{j} x}+\theta\right) \\
& \leq\left(2 \varphi_{0}+\theta\right) \sum_{j=m}^{n-1} 2^{-2(j+1)}+\sum_{j=m}^{n-1} 2^{-2(j+1)} \varphi_{2^{j} x}
\end{aligned}
$$

for all $x \in X$. By (4.1), since the right-hand side of the inequality (4.12) tends to zero as $m$ tends to infinity, the sequence $\left\{g_{n}(x)\right\}$ is a Cauchy sequence for all $x \in X$, and hence we define a function $g: X \rightarrow Y$ by

$$
g(x)=\lim _{n \rightarrow \infty} g_{n}(x) \quad \forall x \in X .
$$

The inequality (4.2) implies that

$$
\begin{aligned}
\| g_{n}\left(2^{n}(x+y+z)\right)+g_{n}\left(2^{n} x\right)+g_{n}\left(2^{n} y\right)+g_{n}\left(2^{n} z\right)-g_{n}\left(2^{n}(x+y)\right) \\
-g_{n}\left(2^{n}(y+z)\right)-g_{n}\left(2^{n}(z+x)\right) \| \leq 2^{-2 n} \varphi\left(2^{n} x, 2^{n} y, 2^{n} z\right)
\end{aligned}
$$

for all $x, y, z \in X$ and $n \in \mathbb{N}$. Letting $n$ tend to infinity in the last inequality, then by (4.1) we obtain (1.3). Analogously, by (4.8), we can see that $g$ is even. By substituting $-y$ for $z$ in (1.3) and by taking account of $g(0)=0$, we see that $g$ as an even solution of (1.3) is quadratic. From (4.3) and (4.8), we have

$$
\begin{aligned}
\left\|f(x)-g_{n}(x)\right\| \leq & \left\|f(x)-\frac{2^{n}+1}{2^{2 n+1}} f\left(2^{n} x\right)+\frac{2^{n}-1}{2^{2 n+1}} f\left(-2^{n} x\right)\right\| \\
& +\left\|2^{-2 n} f\left(2^{n} x\right)-\frac{2^{n}+1}{2^{2 n+1}} f\left(2^{n} x\right)+\frac{2^{n}-1}{2^{2 n+1}} f\left(-2^{n} x\right)\right\| \\
& \leq \sum_{k=1}^{n}\left(\frac{1}{2^{k-1}} \varphi_{0}+\frac{2^{k}+1}{2^{2 k+1}} \varphi_{2^{k-1} x}+\frac{2^{k}-1}{2^{2 k+1}} \varphi_{-2^{k-1} x}\right)+\frac{2^{n}-1}{2^{2 n+1}} \theta
\end{aligned}
$$

for all $x \in X$ and for all $n \in \mathbb{N}$.

According to (4.1) and (4.15), the inequality (4.9) holds true.

The proof of the uniqueness is similar to that of Theorem 2.2 by applying $g\left(2^{n} x\right)=$ $4^{n} g(x)$ and $h\left(2^{n} x\right)=4^{n} h(x)$. Hence, the proof is complete. 
Corollaries 4.3 and 4.5 are the Hyers-Ulam stability of quadratic functional equation (1.3) under the approximately even or odd condition which is the result of Jung [4]. For the proof, apply Theorems 3.5 and 4.2 with $\varphi(x, y, z)=\delta$.

COROLLARY 4.3. Assume a mapping $f: X \rightarrow Y$ satisfies the inequality

$$
\begin{gathered}
\|f(x+y+z)+f(x)+f(y)+f(z)-f(x+y)-f(y+z)-f(z+x)\| \leq \delta, \\
\|f(x)-f(-x)\| \leq \theta,
\end{gathered}
$$

for some $\delta, \theta \geq 0$ and for all $x, y, z \in X$. Then there exists a unique quadratic mapping $g: X \rightarrow Y$ satisfying (1.3) and the inequality

$$
\|g(x)-f(x)\| \leq 4 \delta \quad \forall x \in X .
$$

If, moreover, $f$ is measurable or $f(t x)$ is continuous in $t$ for each fixed $x \in X$, then $g(t x)=t^{2} g(x)$ for all $x \in X$ and $t \in \mathbb{R}$.

THEOREM 4.4. Assume that a mapping $f: X \rightarrow Y$ satisfies the inequalities (4.2) and

$$
\|f(x)+f(-x)\| \leq \theta
$$

for $\theta \geq 0$ and for all $x, y, z \in X$. Then there exists a unique additive mapping $g: X \rightarrow Y$ satisfying (1.1) and

$$
\|g(x)-f(x)\| \leq \Phi(x) \quad \forall x \in X .
$$

If, moreover, $f$ is measurable or $f(t x)$ is continuous in $t$ for each fixed $x \in X$, then $g(t x)=t^{2} g(x)$ for all $x \in X$ and $t \in \mathbb{R}$.

Proof. For any $x \in X$ and for every positive integer $n$, we define

$$
g_{n}(x)=2^{-n} f\left(2^{n} x\right)
$$

From (4.4) and (4.18), we have

$$
\begin{aligned}
& \left\|g_{n+1}(x)-g_{n}(x)\right\| \\
& \quad=2^{-(n+1)}\left\|f\left(2 \cdot 2^{n} x\right)-2 f\left(2^{n} x\right)\right\| \\
& \quad \leq 2^{-(n+1)}\left(\left\|3 f\left(2^{n} x\right)+f\left(-2^{n} x\right)-f\left(2 \cdot 2^{n} x\right)\right\|+\left\|f\left(2^{n} x\right)+f\left(-2^{n} x\right)\right\|\right) \\
& \quad \leq 2^{-(n+1)}\left(2 \varphi_{0}+\varphi_{2^{n} x}+\theta\right)
\end{aligned}
$$

for all $x \in X$ and for all $n \in \mathbb{N}$. Therefore we have, for $n \geq m$,

$$
\begin{aligned}
\left\|g_{n}(x)-g_{m}(x)\right\| & \leq \sum_{j=m}^{n-1}\left\|g_{j+1}(x)-g_{j}(x)\right\| \\
& \leq \sum_{j=m}^{n-1} 2^{-(j+1)}\left(2 \varphi_{0}+\varphi_{2^{j} x}+\theta\right) \\
& \leq\left(2 \varphi_{0}+\theta\right) \sum_{j=m}^{n-1} 2^{-(j+1)}+\sum_{j=m}^{n-1} 2^{-(j+1)} \varphi_{2^{j} x}
\end{aligned}
$$


for all $x \in X$. By definition (4.1) of $\Phi$, since the right-hand side of the inequality (4.22) tends to zero as $m$ tends to infinity, the sequence $\left\{g_{n}(x)\right\}$ is a Cauchy sequence for all $x \in X$, and hence we define a function $g: X \rightarrow Y$ by

$$
g(x)=\lim _{n \rightarrow \infty} g_{n}(x) \quad \forall x \in X .
$$

Similarly, as in the proof of Theorem 4.2, due to (4.18), we see that the mapping $g$ satisfies (1.3) and is odd. By putting $z=-y$ in (1.3) and considering the oddness of $g$ and letting $u=x+y, v=x-y$, we get

$$
2 g\left(\frac{u+v}{2}\right)=g(u)+g(v) .
$$

According to [5], since $g(0)=0$, the mapping $g$ is additive. Similarly-as in the proof of (4.9) of Theorem 4.2-from Lemma 4.1, (4.18), and (4.1), we directly see that (4.19) holds true.

The proof of the uniqueness is similar to that of Theorem 2.2 by applying $g\left(2^{n} x\right)=$ $2^{n} g(x)$ and $h\left(2^{n} x\right)=2^{n} h(x)$. Hence, the proof is complete.

COROLlary 4.5. Assume a mapping $f: X \rightarrow Y$ satisfies the inequality

$$
\begin{gathered}
\|f(x+y+z)+f(x)+f(y)+f(z)-f(x+y)-f(y+z)-f(z+x)\| \leq \delta, \\
\|f(x)+f(-x)\| \leq \theta,
\end{gathered}
$$

for some $\delta, \theta \geq 0$ and for all $x, y, z \in X$. Then there exists a unique additive mapping $g: X \rightarrow Y$ satisfying the inequality

$$
\|g(x)-f(x)\| \leq 4 \delta \quad \forall x \in X .
$$

\section{REFERENCES}

[1] P. W. Cholewa, Remarks on the stability of functional equations, Aequationes Math. 27 (1984), no. 1-2, 76-86. MR 86d:39016. Zbl 549.39006.

[2] S. Czerwik, On the stability of the quadratic mapping in normed spaces, Abh. Math. Sem. Univ. Hamburg 62 (1992), 59-64. MR 94e:39026. Zbl 779.39003.

[3] P. Găvruța, A generalization of the Hyers-Ulam-Rassias stability of approximately additive mappings, J. Math. Anal. Appl. 184 (1994), no. 3, 431-436. MR 95e:47089. Zbl 818.46043.

[4] S.-M. Jung, On the Hyers-Ulam stability of the functional equations that have the quadratic property, J. Math. Anal. Appl. 222 (1998), no. 1, 126-137. MR 99e:39095. Zbl 928.39013.

[5] J. C. Parnami and H. L. Vasudeva, On Jensen's functional equation, Aequationes Math. 43 (1992), no. 2-3, 211-218. MR 93e:39013. Zbl 755.39008.

[6] T. M. Rassias, On the stability of the linear mapping in Banach spaces, Proc. Amer. Math. Soc. 72 (1978), no. 2, 297-300. MR 80d:47094. Zbl 398.47040.

[7] F. Skof, Local properties and approximation of operators. Geometry of Banach spaces and related topics (Italian) (Milan, 1983), Rend. Sem. Mat. Fis. Milano 53 (1983), 113-129 (1986) (Italian). MR 87m:39009. Zbl 599.39007.

[8] S. M. Ulam, Problems in Modern Mathematics, Science Editions, John Wiley \& Sons, Inc., New York, 1964. MR 43\#6031. Zbl 137.24201.

GWANG Hui Kim: Department of Mathematics, KANGNAm UniVersity, SuWON, 449-702, KOREA

E-mail address: ghkim@kns.kangnam.ac.kr 


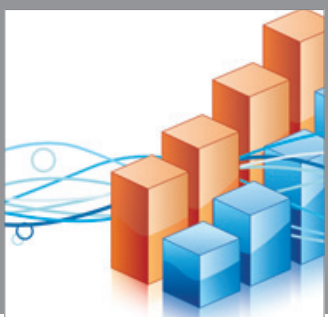

Advances in

Operations Research

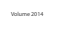

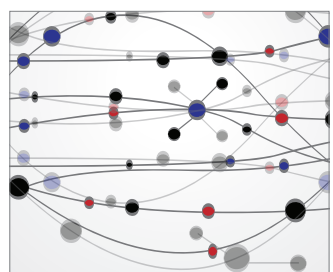

\section{The Scientific} World Journal
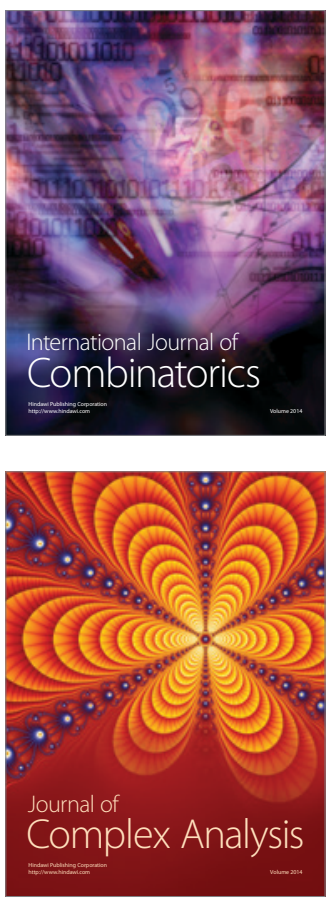

International Journal of

Mathematics and

Mathematical

Sciences
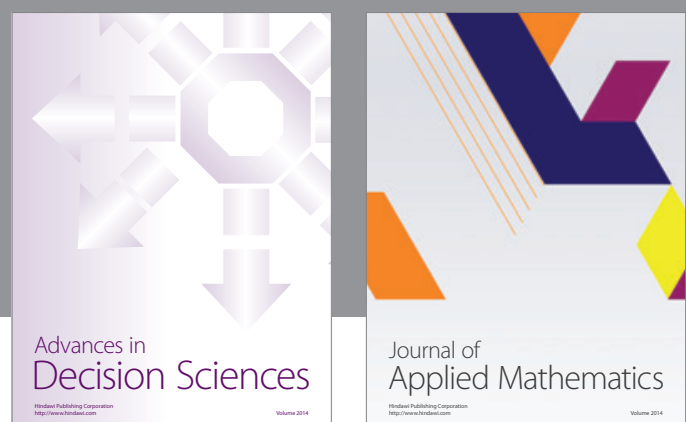

Journal of

Applied Mathematics
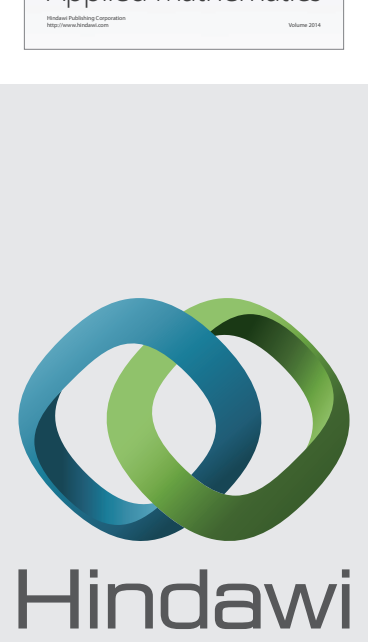

Submit your manuscripts at http://www.hindawi.com
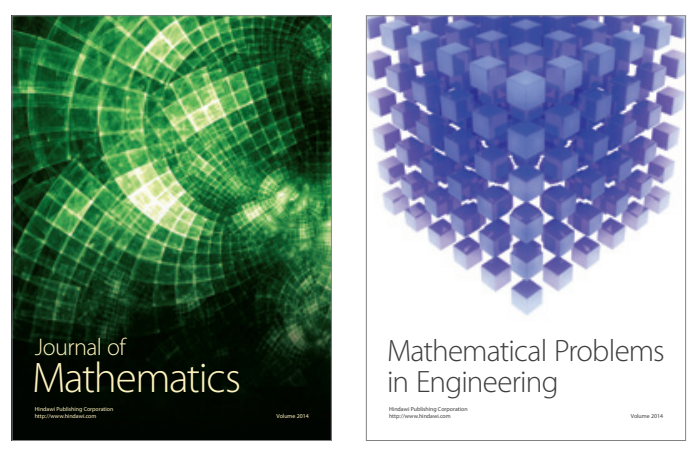

Mathematical Problems in Engineering
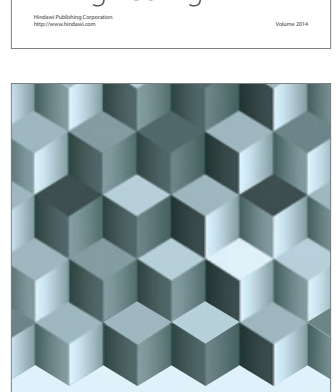

Journal of

Function Spaces
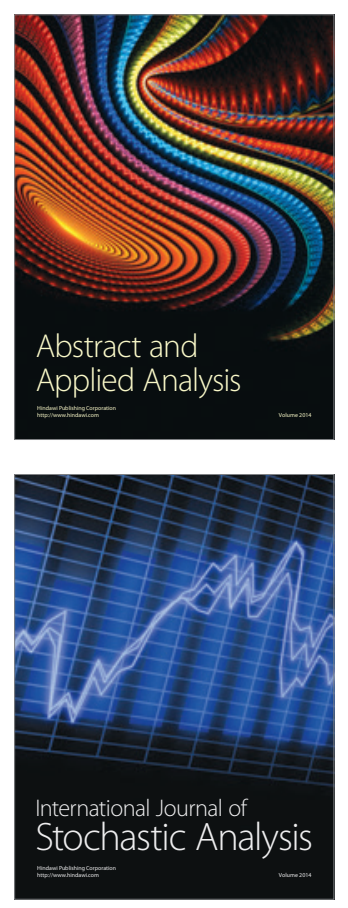

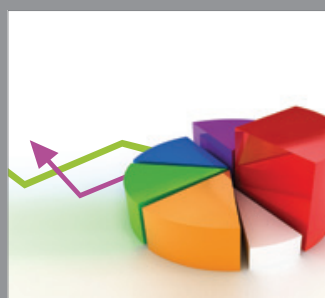

ournal of

Probability and Statistics

Promensencen
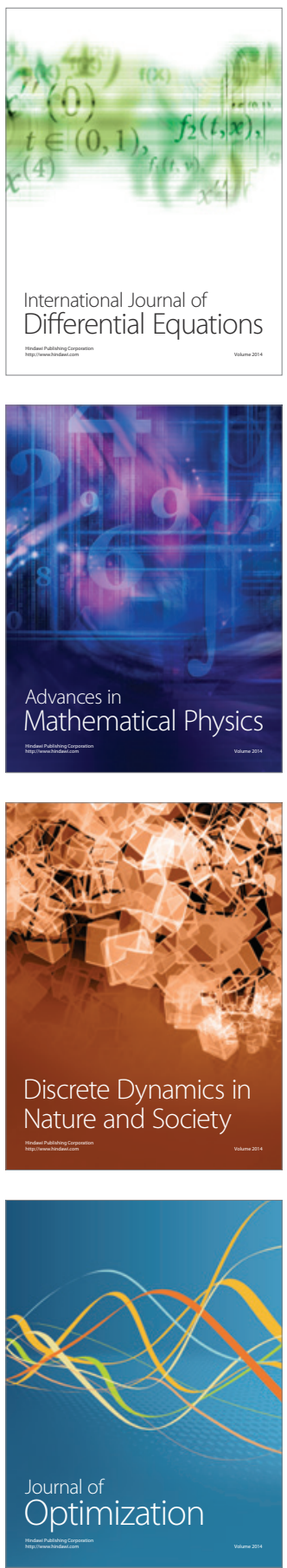